University of Louisville

ThinkIR: The University of Louisville's Institutional Repository

\title{
Filifactor alocis, a newly appreciated oral pathogen, fails to induce the respiratory burst response of human neutrophils.
}

Jacob S. Edmisson

Follow this and additional works at: https://ir.library.louisville.edu/honors

Part of the Biology Commons, Oral Biology and Oral Pathology Commons, and the Periodontics and Periodontology Commons

\section{Recommended Citation}

Edmisson, Jacob S., "Filifactor alocis, a newly appreciated oral pathogen, fails to induce the respiratory burst response of human neutrophils." (2016). College of Arts \& Sciences Senior Honors Theses. Paper 109.

http://doi.org/10.18297/honors/109

This Senior Honors Thesis is brought to you for free and open access by the College of Arts \& Sciences at ThinkIR: The University of Louisville's Institutional Repository. It has been accepted for inclusion in College of Arts \& Sciences Senior Honors Theses by an authorized administrator of ThinkIR: The University of Louisville's Institutional Repository. This title appears here courtesy of the author, who has retained all other copyrights. For more information, please contact thinkir@louisville.edu. 


\section{Filifactor alocis, a newly appreciated oral pathogen, fails to induce the respiratory burst response of human neutrophils}

By

Jacob S. Edmisson

Submitted in partial fulfillment of the requirements for Graduation summa cum laude

and

for Graduation with Honors from the Department of Biology

University of Louisville

March, 2016 


\section{Table of Contents}

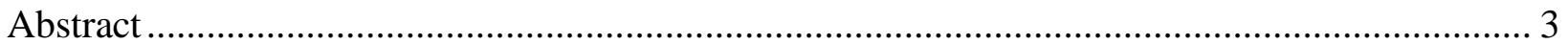

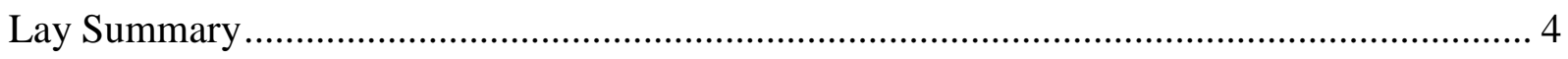

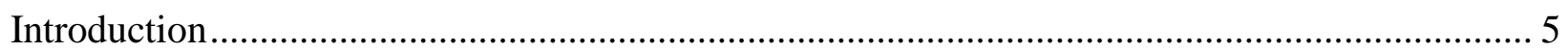

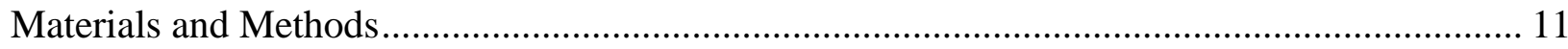

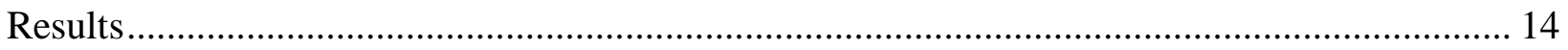

F. alocis fails to induce neutrophil respiratory burst response.............................................. 14

Products secreted during $F$. alocis growth primed neutrophil respiratory burst response........ 17

F. alocis challenge neither primed nor inhibited neutrophil respiratory burst response .......... 19

Viable $F$. alocis, but not heat-killed bacteria, delays specific granule recruitment to bacteria-

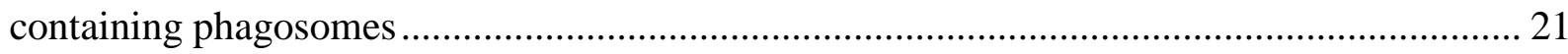

F. alocis induced secretory vesicle and specific granule exocytosis ...................................... 24

F. alocis induced minimum gelatinase granule exocytosis ................................................... 26

F. alocis does not induce azurophil granule exocytosis ...................................................... 27

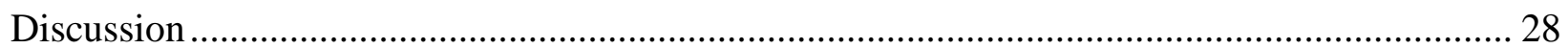

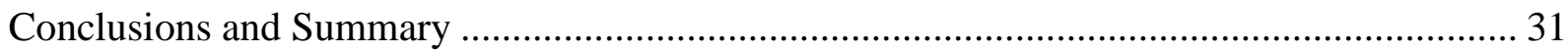

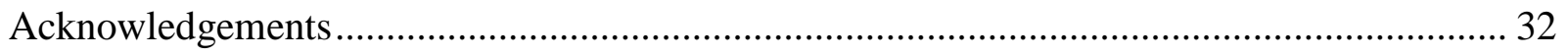

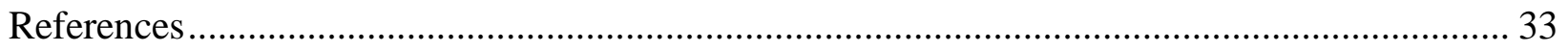




\section{Abstract}

Almost 50\% of adult Americans suffer from periodontitis which is a bacterially induced inflammation of the tissue that surround and support the tooth. The accumulation of neutrophils, a critical cell component of the innate immune system, in the gingival crevice contributes to tissue damage. Filifactor alocis is a newly appreciated pathogen present in oral biofilms at periodontal disease sites. Studying the interactions between neutrophils and F. alocis will provide valuable information for delineating the role of this bacterium in periodontal disease and enhance our understanding of bacterial strategies to evade leukocytes' antimicrobial mechanisms. The hypothesis that $F$. alocis modulates human neutrophil antimicrobial functions was tested. One of several antimicrobial mechanisms employed by the neutrophil is the respiratory burst response with production of reactive oxygen species within bacteria-containing phagosomes. Previous studies in our lab showed that human neutrophils challenged for $30 \mathrm{~min}$ with either non-opsonized or serum opsonized $F$. alocis at a multiplicity of infection (MOI) of 10 failed to induce a robust respiratory burst response. In addition, serum opsonized $F$. alocis failed to induce the respiratory burst response even after 60 and $120 \mathrm{~min}$ post neutrophil challenge. Interestingly, neutrophils challenged for 30 min with the serum opsonized heat-killed organism at an MOI of 10 elicited a 2.5 fold higher intracellular respiratory burst response compared to viable $F$. alocis. To determine if the failure to induce the respiratory burst was mediated by secreted bacterial products, neutrophils were exposed to the $F$. alocis culture supernatant and the oxidative burst response was determined. The culture supernatant by itself did not induce a respiratory burst response; however, it primed the S. aureus-stimulated response. The recruitment of specific granules to the phagosome assists in the accumulation of NADPH oxidase complexes because they contain approximately $60 \%$ of the membrane-bound subunits; 
therefore, a possible strategy to avoid/delay killing would be to hinder specific granule recruitment post-phagocytosis. After $30 \mathrm{~min}$ of bacteria challenge, only $35 \%$ of viable $F$. alociscontaining phagosomes were enriched for lactoferrin positive granules, compared to 66-70\% of heat-killed F. alocis or $S$. aureus-containing phagosomes. Diverting granule exocytosis to the plasma membrane instead of the bacterial phagosome could prevent bacterial killing and contribute to the tissue damage characteristic of periodontitis. Serum opsonized F. alocis induced significant secretory vesicle and specific granule exocytosis; whereas the heat-killed bacteria significantly reduced secretory vesicle exocytosis but stimulated specific granule exocytosis to the same extent as the viable bacteria. F. alocis also induced gelatinase granule exocytosis, but did not cause azurophil granule release. These data demonstrate previously unexplored aspects of the new oral pathogen $F$. alocis and how this species modulates neutrophil function. By examining the differences between viable and heat-killed $F$. alocis and the effector molecules released by the pathogen during growth, I will begin to characterize $F$. alocis' role in the pathogenesis of periodontitis.

\section{Lay Summary}

Periodontal disease affects numerous people worldwide, and it is correlated with many other inflammatory diseases. This disease is caused by an imbalance between the oral bacterial community and the host immune system. Neutrophils are white blood cells that regularly enter the gingival tissues to keep the bacteria populations under control. In periodontal disease, the neutrophils add to the tissue damage without resolving the infection. One of the species, Filifactor alocis has recently been recognized. To determine its role in the disease, F. alocis is studied with human neutrophils. In this study, I found the $F$. alocis inhibits neutrophil killing mechanisms while simultaneously increasing release of certain tissue damaging agents. 


\section{Introduction}

Periodontal disease is a poly-microbial induced disease caused by the accumulation of pathogenic bacteria which outcompete the commensal microbes in the oral cavity (1). Periodontal problems are among the most common reasons for doctor visits for both children and adults especially with poor diet or low socioeconomic status $(2,3)$. In fact, it has been estimated that over $40 \%$ of Americans will experience some form of periodontal disease (3). Specifically, periodontitis is a chronic infection which results in inflammation of the periodontium (the gingiva, periodontal ligament, cementum and alveolar bone). Treatment includes deep cleaning, antibiotics and surgery in severe cases (4). Unfortunately, these treatments are only efficacious in the short term because the infection almost always returns (4). This disease has also been associated with several comorbidities including rheumatoid arthritis and cardiovascular disease among other inflammatory conditions (4-6). Due to recent improvements in sequencing technology, new organisms have been recognized as a potential indicator of periodontal disease. These organisms appear at high percentage levels in the oral cavity in victims of periodontal disease and are almost absent in healthy mouths (2).

The current model describing the etiology and pathogenesis for periodontitis is the polymicrobial synergy and dysbiosis (PSD) model $(1,7)$. The PSD model indicates that an imbalance between commensals and pathogenic bacteria results in a cyclic inflammation that clears beneficial bacteria while facilitating the growth of the pathogens (7). Unlike the classical infection which is cleared by inflammation, these pathogens induce the associated tissue damage to gain valuable nutrients (8). On the side of the pathogens, there is a delicate interplay between keystones and pathobionts (1). Pathobionts are similar to opportunistic pathogens because they 
take advantage of a diseased state. However, unlike opportunistic infections, these pathogens already colonize the area. They are just able to proliferate during the inflammation. For years, the studies around periodontal disease have focused on what has been called the "red complex" named for the redness of the inflammation associated with the disease $(1,2,7)$. The red complex includes three keystones organisms which are thought to be necessary for disease onset: Porphyromonas gingivalis, Tannerella forsythia, and Treponema denticola (7). Among these, $P$. gingivalis has been the most extensively studied. However, $P$. gingivalis is not sufficient to cause infection in germ-free animal models (9). Pathobionts are the other critical component for establishment of infection and eventual disease (1). These are organisms which exist in the oral microbiota, but opportunistically take advantage of the inflammation (8). Pathobionts ameliorate the environment for other periopathogens and contribute to manipulation of host immunity to propagate inflammation (1).

The newly cultivated bacteria Filifactor alocis has been proposed as one of these pathobionts due to its high concentration in periodontal disease patients compared to healthy mouths (10). Interestingly, F. alocis is found in higher quantities than P. gingivalis which has led to it being proposed as a diagnostic indicator of the disease (11). F. alocis is a gram-positive rodshaped asaccharolytic (uses amino acids as its carbon source) bacteria that is slow growing and difficult to plate (10). This species also shares common characteristics with other periopathogens such as a resistance to oxidative stress, evasion of host immune system, biofilm formation and proteases which may contribute to tissue damage $(10,11)$. Previous studies in $F$. alocis' role in biofilm formation have also shown that it facilitates $P$. gingivalis growth (12). Its ability to cause disease may not be solely restricted to the periodontal pocket. Mouse models have also shown 
the bacteria can spread to other tissues such as spleen, lung and kidney tissues with acute kidney damage observed resulting from inflammation (13).

The first immune cell to respond and be recruited in vast numbers to the site of infection is the neutrophil $(14,15)$. These cells are professional phagocytic cells involved in the innate branch of the immune system (16). They possess numerous strategies to locate, detain and kill microbes $(16,17)$. Neutrophils have multiple killing mechanisms to eliminate both intracellular as well as extracellular microorganisms (16). If the neutrophil can then its first attempt to clear infection will be phagocytosis of the infectious microbes. The intracellular killing mechanisms are dependent upon the internalization of the microbe. Upon encounter with the offending microbe the phagocytic process is initiated followed by high oxygen consumption through a process known as the respiratory burst. The oxygen is used in the generation of reactive oxygen species (ROS) within the bacteria-containing phagosome (18). In addition, maturation of the phagosome is achieved by the recruitment of neutrophil granules to the bacteria-phagosome which does not involve the consumption of oxygen and is described as a neutrophil oxygenindependent antimicrobial mechanism (18). The process of phagosome maturation is typically a quick process that is correlated with the time necessary to mount the respiratory burst (18).

To combat extracellular microorganisms, which resist or evade phagocytosis, neutrophils will mount both an extracellular oxygen-dependent killing response with the generation and release of oxygen radicals into the external environment. They will also utilize an oxygenindependent killing response by exocytosing anti-microbial contents held within the neutrophil's granules through a process known as exocytosis $(17,19)$.

The last antimicrobial weapon available to neutrophils is the production of neutrophil extracellular traps or NETs (17). If the offending agent is too large for the neutrophil to 
phagocytose and is not quickly cleared by granule exocytosis then NETs are considered a last effort to detain the microbe (20). Some bacteria can also induce NETosis, and NETs are found in high quantities in the crevicular fluid of periodontal disease patients (21).

The buccal cavity is constantly filled with microbes. So, neutrophils are continuously entering these tissues (15). Neutrophils respond to the infection/bacteria burden by leaving the blood vessels and entering the periodontal pocket with the goal of destroying the offending microbes to maintain homeostasis in the oral cavity (17). In periodontal disease, the periodontal pathogens have the ability to break the balance between the immune system and the microbiota perpetrating a chronic inflammatory environment which benefits the oral pathogenic community $(1,15)$. This results in profuse accumulation of both neutrophils and pathogens (8). The neutrophils are activated but unable to clear the infection and instead their anti-microbial weapons are turned on the host $(15,19,22)$. For instance, neutrophils possess four different types of granules whose contents can be release through exocytosis (as described above) to both antimicrobial and tissue damaging ends (19). These granules are the azurophil, specific, gelatinase granules and the secretory vesicles (Table 1). In this way, the pathogens use the host's own immune system to their benefit. 
Table 1

\begin{tabular}{|c|c|c|c|c|}
\hline $\begin{array}{l}\text { Granule } \\
\text { Subtype }\end{array}$ & Membrane Contents & Matrix Contents & $\begin{array}{c}\text { Degranulation } \\
\text { Propensity }\end{array}$ & $\begin{array}{l}\text { Formation } \\
\text { Timing }\end{array}$ \\
\hline $\begin{array}{c}\text { Azurophil } \\
\text { (primary) } \\
\text { granules }\end{array}$ & N. a. & $\begin{array}{c}\text { Elastase, } \\
\text { Cathepsin G } \\
\text { Proteinase } 3 \\
\text { Myeloperoxidase } \\
\text { a-Defensins } \\
\text { BPI } \\
\text { Lysozyme }\end{array}$ & Very low & First \\
\hline $\begin{array}{c}\text { Specific } \\
\text { (secondary) } \\
\text { granules }\end{array}$ & Gp91phox/p22phox & $\begin{array}{c}\text { Collagenase } \\
\text { Gelatinase } \\
\text { uPA } \\
\text { Cystatin C/F } \\
\text { hCAP18 } \\
\text { NGAL } \\
\text { B12BP } \\
\text { Lysozyme } \\
\text { Lactoferrin } \\
\text { Haptoglobin } \\
\text { Pentraxin 3 } \\
\text { Prodefensin }\end{array}$ & Moderate & Second \\
\hline $\begin{array}{c}\text { Gelatinase } \\
\text { (tertiary) } \\
\text { granules }\end{array}$ & $\begin{array}{c}\text { Gp91phox/p22phox } \\
\text { MMP25 }\end{array}$ & $\begin{array}{l}\text { Gelatinase } \\
\text { Arginase } 1 \\
\text { Lysozyme }\end{array}$ & High & Third \\
\hline $\begin{array}{l}\text { Secretory } \\
\text { Vesicles }\end{array}$ & $\begin{array}{c}\text { Gp91phox/p22phox } \\
\text { MMP25 }\end{array}$ & N. a. & Very High & Final \\
\hline
\end{tabular}

Table 1: Neutrophil granule subtypes' contents, formation and exocytosis potential. Each granule subtype is listed along

with any microbicidal or protease contents which are divided between those on the granule membrane or within the granule's lumen (matrix). The propensity for exocytosis and the order in which the granules are formed are also included.

This list is non-exhaustive and this table is adapted from previous reviews $(17,19)$.

One of the most critical anti-microbial mechanisms that neutrophil possess is the induction of the oxygen-dependent or respiratory burst response, where activation of the NADPH oxidase complex results in high and rapid oxygen consumption and generation of oxygen radicals $(17,18,23)$. If neutrophils encounter a soluble stimuli, the NADPH oxidase complex will assemble at the cell plasma membrane and the oxygen radicals will be generated extracellularly; however, if the cells encounter a particulate stimuli, like a bacterium, the oxidase 
complex will be activated in the phagosome membrane and oxygen radicals release inside that structure with limited tissue damage (18). Upon phagocytosis of a bacterium, this enzymatic NADPH oxidase complex assembles on the phagosome and produces the oxygen radicals which are highly damaging to macromolecules such as protein and DNA and will compromise the bacterium growth $(23,24)$. A common bacterial strategy to survive the oxygen-dependent antimicrobial attack from the neutrophils is the inhibition, evasion or neutralization of the NADPH oxidase $(22,25)$.

Previous studies have examined the interplay between the host immune system and periopathogens (15). However, no work has yet looked into the relationship between $F$. alocis and human neutrophils. Previous studies in our lab have shown that $F$. alocis can survive 6+ hours in the neutrophil post-phagocytosis which indicates that neutrophils are inefficient to kill this oral emerging pathogen. It is possible that either inhibition of the respiratory burst response or failure to activate it contributes to $F$. alocis' remarkable survival time. To begin to define how the emerging oral bacterium is modulating neutrophil anti-microbial functions, I have challenged human neutrophils with both viable and heat-killed $F$. alocis. I also begin to examine the effects of secreted products of $F$. alocis' metabolism on the intracellular respiratory burst response. My working hypothesis is that $F$. alocis manipulates neutrophil killing mechanisms to promote inflammation. 


\section{Materials and Methods}

\section{a) Human neutrophil isolation.}

Neutrophils were isolated from healthy human donors using plasma-Percoll gradients as previously described (26). Microscopic evaluations using Wright staining (ENG Scientific, Inc) show that $>90 \%$ of isolated cells were neutrophils for each experiment that will be used. The isolated cell fraction was mounted onto a slide using centrifugation (CytoSpin) and then fixed in methanol. Wright staining utilizes eosin to stain the cytoplasm and methylene blue to stain nuclei. A hemocytometer was used to count the number of neutrophils relative to other blood cells such as eosinophils or basophils to establish the percentage of cell purity.

\section{b) F. alocis culture.}

F. alocis strains were cultured anaerobically at $37^{\circ} \mathrm{C}$ in $\mathrm{BHI}$ supplemented with hemin, menadione and arginine as previously described (13). Bacteria were killed by heat after incubation at $90^{\circ} \mathrm{C}$ for $1 \mathrm{hr}$.

\section{c) Intracellular respiratory burst assay.}

Phagocytosis-stimulated respiratory burst was measured by oxidation of 2', 7 'dichlorofluorescin diacetate (DCF) [Molecular Probes/ Invitrogen, Carlsbad, CA] analyzed by flow cytometry using a BD FACSCalibur ${ }^{\mathrm{TM}}(18,27)$. Each condition of $1 \mathrm{ml}$ solutions of isolated human neutrophils $\left(4 \times 10^{6}\right.$ cells $\left./ \mathrm{ml}\right)$ with $5 \mu \mathrm{M}$ final concentration of DCF were incubated together for 10 minutes. After incubation with DCF, $500 \mu$ l aliquots were removed and kept on ice for analysis. These aliquots are referred to as time-zero (T0) samples and were washed in FTA buffer with $0.05 \% \mathrm{NaN}_{3}$ and fixed in FTA buffer with $1 \%$ paraformaldehyde. T0 samples are used as the negative control for the respiratory burst response in which cells have not received any stimulation yet. Remaining cells after 10 min of DCF treatment were challenged 
with different stimuli to induce the respiratory burst response. Those stimulated samples for 30 min were labeled as T30. In some experimental designs cells were pre-treated with the bacteria spent supernatants and in those conditions DCF was added after the pre-treatment period.

Samples used in experiments for testing the spent supernatants used filtered broth (as described in "methods e") and all other experiments used $\mathrm{Krebs}^{+}$buffer. Readings from T0 samples were used to normalize the experimental conditions. All samples are analyzed using a BD FACSCalibur $^{\mathrm{TM}}$.

\section{d) Granule recruitment to bacteria-containing phagosomes.}

Neutrophils $\left(1 \times 10^{6}\right.$ cells/coverslip $)$ were allowed to attach to serum coated coverslips for 30 min before stimulation. After a 30 min incubation time, cells were challenged with carboxyfluorescin succinimidyl ester (CFSE)-labeled $F$. alocis, or heat-killed CFSE-labeled $F$. alocis, or Alexa Fluor 488-labeled S. aureus at an MOI of 10:1 for 30 minutes. Attached neutrophils were then washed, permeabilized and fixed. After infection, polyclonal antibodies (MP Biomedicals) for lactoferrin (an iron chelator uniquely found in specific granules), or elastase (a protease found only in azurophil granules), was allowed to incubate with the cells followed by a labeled secondary antibody (Alexa Fluor 555 goat anti-rabbit [Life Technologies]). To visualize the cell nucleus DAPI (4', 6-diamidino-2-phenylindole) was added to the coverslips for $5 \mathrm{~min}$. This method was adapted from a previously described protocol (28). Confocal images were taken using an Olympus FV1000 confocal microscope. Serial sections of $10 \mu \mathrm{m}$ (Z-stacks) of the infected neutrophils were used to determine specific granule or azurophil granule fusion to bacteria-containing phagosomes. 100 infected cells were counted per experiment and phagosomes were considered positive for the granule marker if more than $50 \%$ of the bacteria were surrounded by it. 


\section{e) Spent Supernatant Preparation.}

F. alocis cultures as described in "methods b" were centrifuged, and the supernatant from their growth was collected. To remove bacteria residues from the growth culture but preserve the proteins of interest, the supernatant collected were filtered through a sterile ultra-low protein binding filter (Millex-GV Syringe Filter Unit, $0.22 \mu \mathrm{m}, \mathrm{PVDG}, 33 \mathrm{~mm}$, gamma sterilized).

\section{f) Neutrophil granule exocytosis.}

Isolated human neutrophils $\left(4 \times 10^{6}\right.$ cells $\left./ \mathrm{ml}\right)$ were suspended in $\mathrm{Krebs}^{+}$buffer and were unstimulated (negative control), or stimulated with fMLF (300 nM, 5 min, [Sigma, St. Louis, MO]), a formylated bacteria peptide used as a positive control, or TNF- $\alpha+$ fMLF (a positive control for azurophil granule exocytosis only), or challenged with $F$. alocis (multiplicity of infection (MOI) 10 bacteria per neutrophil (10:1, for 30 minutes) at $37^{\circ} \mathrm{C}$. The exocytosis of azurophil granules, specific granules, and secretory vesicles was determined by measuring the increase in the presence of fluorescently tagged granule markers (CD63, CD66b, and CD35 respectively) on the cell plasma membrane with a FACS Calibur flow cytometer as previously described $(29,30)$. After stimulation, cells were incubated on ice in the dark for 45 minutes with fluorochrome-conjugated antibodies specific to each granule marker. These were fluoresecein isothiocyanate (FITC)-conjugated anti-CD63 (azurophil granule, Ancell 215-040, Stillwater, MN, USA), FITC-conjugated anti-CD66b (specific granule, Biolegend 305104, San Diego, CA, USA), and phycoerythrin (PE)-conjugated anti-CD35 (secretory vesicle, Biolegend 333406, San

Diego, CA, USA). Following antibody incubation, cells were washed with $0.05 \% \mathrm{NaN}_{3}(\mathrm{~S} 2002$, Sigma, St. Louis, MO, USA) in FTA buffer (211248 BD, Franklin Lakes, NJ, USA) and fixed with 1\% paraformaldehyde (PX0055-3, EMD, Darmstadt, Germany). 
Exocytosis of gelatinase granules was determined by measuring the amount of matrix metalloprotease (MMP)-9 present in the supernatants of unstimulated neutrophils $\left(4 \times 10^{6}\right.$ cells/ml), or fMLF-stimulated (300 nM, $5 \mathrm{~min}$ ), or F. alocis-stimulated; via a commercially available enzyme-linked immunosorbent assay (ELISA, R\&D Systems, Minneapolis, MN, USA). For supernatant sample collection, the supernatants were mixed with $1 \%$ phosphatase and $1 \%$ protease inhibitors and stored in sterile microcentrifuge tubes at $-80^{\circ} \mathrm{C}$ until use.

\section{g) Statistical analysis.}

Statistical differences among experimental conditions and time points were analyzed by one-way analysis of variance (ANOVA) and the post-hoc Tukey multiple-comparison or by twotailed Student's t-test using GraphPad Prism Software (Graphpad San Diego, CA, USA). Differences were considered significant at the level $\mathrm{P}<0.05$.

\section{Results}

\section{F. alocis fails to induce neutrophil respiratory burst response.}

Inhibition or evasion of the respiratory burst response is a very common mechanism for bacteria and other microbes to avoid neutrophil killing (22). To begin to define neutrophil antimicrobial mechanisms against the oral bacterium, I first examine the respiratory burst response induced by phagocytosis of $F$. alocis and compared it to the robust response induced by phagocytosis of Staphylococcus aureus. Both S. aureus and F. alocis were opsonized with normal human serum and used at a multiplicity of infection (MOI) of 10 bacteria per cell. This multiplicity of infection was chosen because it is sufficient to show the respiratory burst response in the positive control (S. aureus) and because it provides a quantifiable number of bacteria per cell in later microscopy experiments. The opsonization process coats the bacteria with serum 
proteins which facilitate their internalization by professional phagocytic cells like neutrophils. To establish if $F$. alocis challenge would trigger neutrophil respiratory burst response, neutrophils were challenged with opsonized $F$. alocis for 30- 60-120 min (Fig 1). No time dependent increase in respiratory burst response was observed when neutrophils were challenged with $F$. alocis relative to basal levels (Fig 1). As expected, S. aureus induced a robust and significant respiratory burst response (Fig 1). These results show that $F$. alocis fails to induce a robust respiratory burst response even after long infection times. As previous stated, the respiratory burst response is generally very quick after phagocytosis. However, these results show that the bacteria are not inducing the response even at 120 min post-infection.

\section{Figure 1}

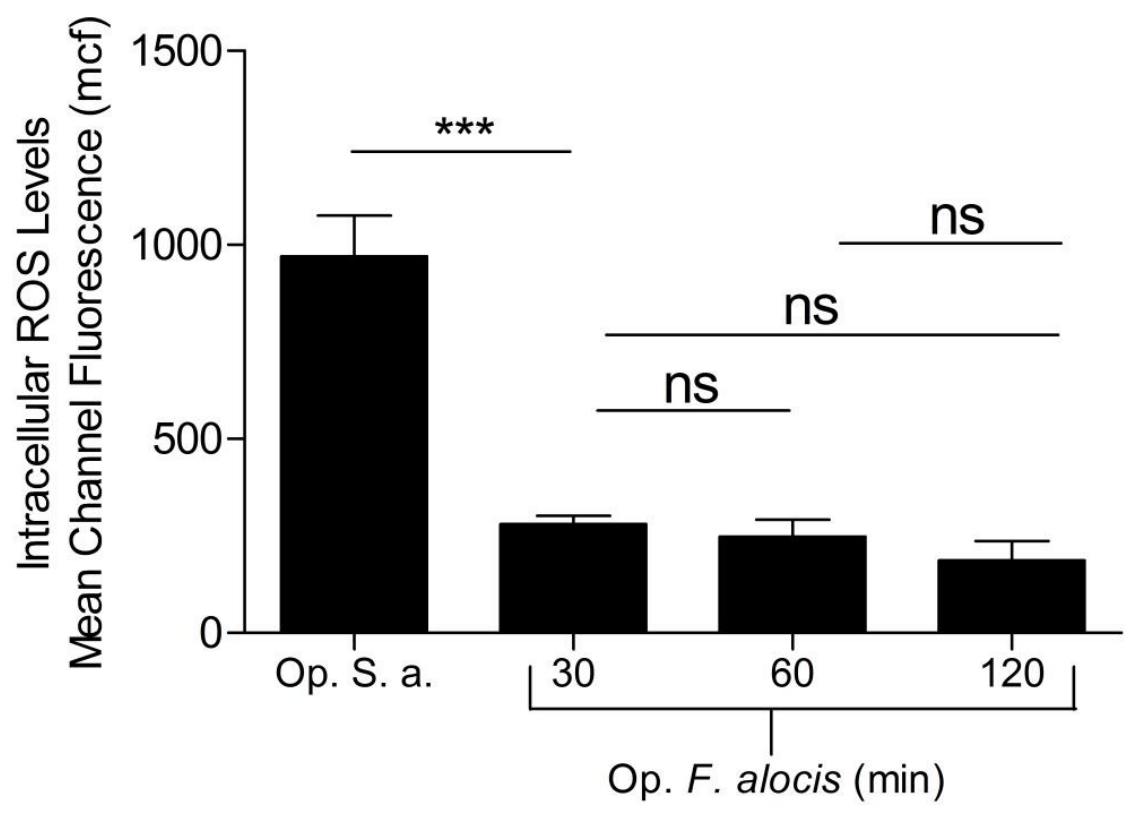

Figure 1: Filifactor alocis fails to induce neutrophil respiratory burst response after 2 hr of neutrophil challenge. Human neutrophils were incubated with serum opsonized Staphylococcus aureus (Op. S. a.; 30 min), or 10\% human serum opsonized Filifactor alocis (Op. F. alocis) for 30-60-120 min. All conditions used a multiplicity of infection (MOI) of 10:1. Data are expressed as mean \pm SEM of the mean channel of fluorescence $(\mathbf{m c f}), \mathbf{n}=\mathbf{4}$ independent experiments. $* * *=$ p<0.001; ns= no significance by one-way ANOVA. 
Since no significant change in ROS levels were observed over time, further investigations of respiratory burst response were performed at $30 \mathrm{~min}$ infections. To determine if the oral bacteria were actively inhibiting the respiratory burst response, neutrophils were challenged with op-F. alocis, or op-heat-killed $F$. alocis. The heat-killed bacteria are disrupted due to protein denaturing. Interestingly, these data show that heat-killed $F$. alocis induced a robust respiratory burst response which was significantly higher than the response induced by the viable bacterium (Fig 2). This difference suggests that inhibition of the oxidase could be protein or metabolite mediated. Moreover, the robust response induced by the heat-killed F. alocis was significantly higher than the positive control used for this assay $S$. aureus. These results indicate that viable $F$. alocis is able to manipulate and prevent the respiratory burst response.

\section{Figure 2}

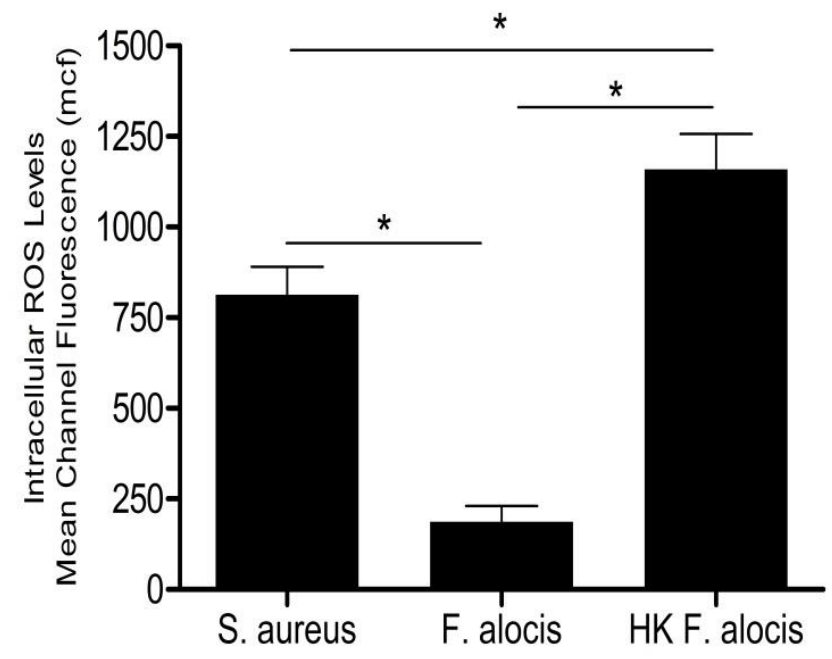

Figure 2: Heat-killed $F$. alocis induces a very robust respiratory burst response. Human serum opsonized $S$. aureus, or opsonized viable $F$. alocis ( $F$. alocis), or opsonized heat-killed $F$. alocis (HK $F$. alocis) were incubated with human neutrophils at an MOI of 10:1 for $30 \mathrm{~min}$. Data are expressed as mean \pm SEM of mean channel of fluorescence (mcf), $n=4$ independent experiments. ${ }^{*}=p<0.05$ by one-way ANOVA. 


\section{Products secreted during $F$, alocis growth primed neutrophil respiratory burst response}

My observations between the viable and heat-killed bacteria led us to further examine the question of how $F$. alocis is inhibiting the respiratory burst response. Since only the viable bacteria are capable of preventing ROS generation, I hypothesized that secreted products of $F$. alocis' own metabolism were responsible for the effects previously observed. To examine the possible inhibitory effects of the secreted products, I prepared spent supernatants. The preparation of these supernatants is summarized in figure 3A. I then incubated neutrophils in these supernatants for $15 \mathrm{~min}$ prior to a $30 \mathrm{~min}$ infection with either F. alocis or S. aureus. If the effects of the supernatants were inhibitory then the robust respiratory burst response seen in $S$. aureus would be reduced. As controls, I tested the neutrophils response to the growth media alone. This clean media labeled "Broth" in figure 3B was also tested against the krebs + media used for other intracellular ROS assays, and they were found to be nearly identical (data not shown).

The spent supernatants were classified in two different categories to assess possible concentration effects. The growth or "G sup" condition uses the media from the full 1-2 weeks necessary for the bacteria to grow to usable levels. The overnight or "ON sup" indicates the media in which the bacteria were incubated for 24 hours (Fig 3A). These supernatants were also tested on their own to assess for possible activity. 


\section{Figure 3}

A.

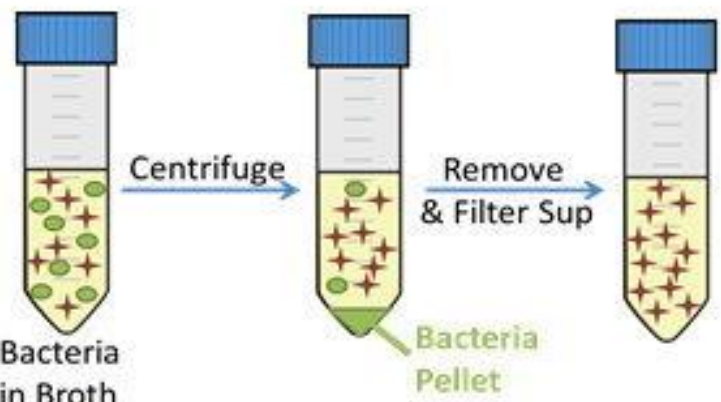

in Broth

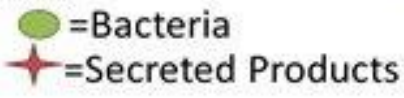

B.

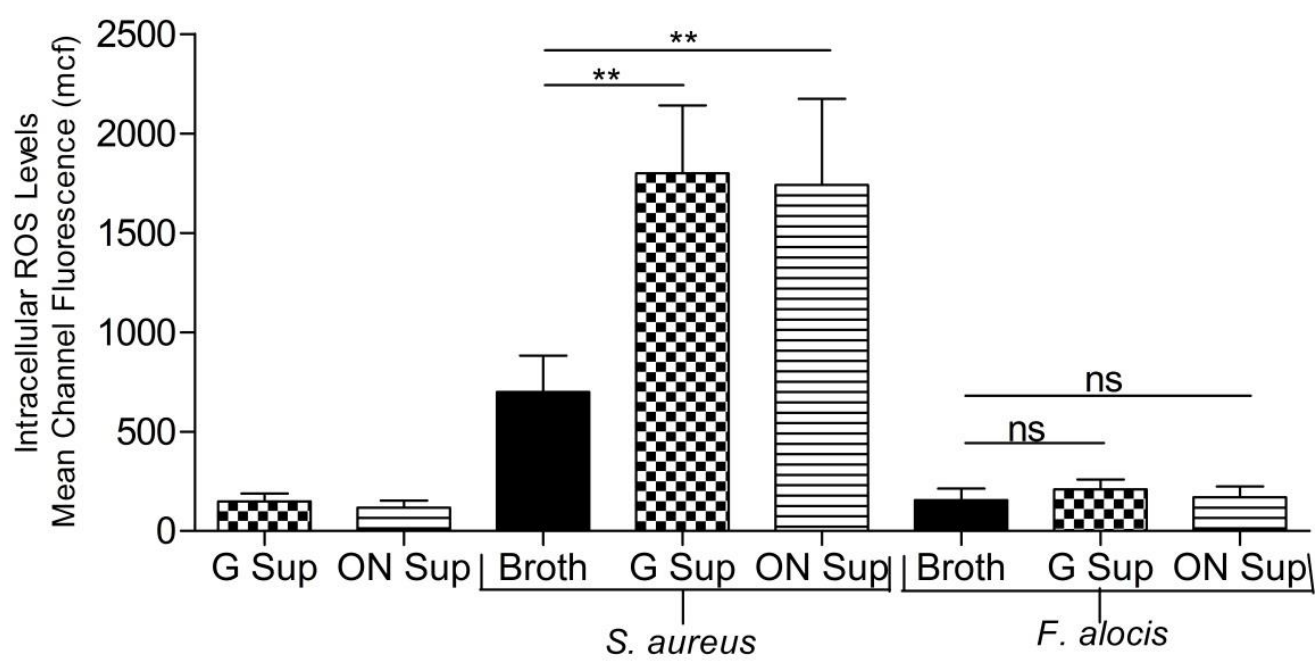

Figure 3: Spent supernatants prime neutrophils. Bacterial growth supernatants (spent supernatants) were collected and used as pretreatment for $15 \mathrm{~min}$. Growth supernatant ( $\mathrm{G}$ sup) is collected after the normal growth period of 8-14 days while overnight (ON Sup) is collected after 24 hrs culture. Following the pretreatment, opsonized S. aureus or opsonized F. alocis were added for $30 \mathrm{~min}$ infections. All bacterial conditions used an MOI of 10:1. Data are expressed as mean \pm SEM of mean channel of fluorescence, $\mathrm{n}=5$ independent experiments. $* *=p<0.01$; $\mathrm{ns}=$ no significance by one-way ANOVA

During an inflammatory setting in vivo, quiescent neutrophils can change into a preactivated phenotype or primed cell due to exposure to inflammatory cytokines such as TNF- $\alpha$, 
interleukin (IL)-1, IL-1 $\beta$ and IL-17 (31, 32). Priming allows the neutrophil to have a more robust response when encountering a second stimulus. A priming agent, such as TNF- $\alpha$, by itself does not result in generation of oxidants, but will induce a robust respiratory burst response when the cells are challenge with a second stimulus like a bacterium in our experiments (32). Interestingly, our data showed that the supernatants collected from $F$. alocis growth culture, exhibited a priming response to the neutrophils as opposed to an inhibitory one (Fig 3B). Our data showed that the supernatants on their own induce minimal oxidant production, but when cells were challenged with a second stimulus like $S$. aureus a significant respiratory burst response was observed (Fig 3B).

However, the priming effect induced by the spent supernatants is muted when F. alocis is used as the second stimulus (Fig 3B). With or without the supernatants pre-treatment, F. alocis still fails to initiate a robust respiratory burst response. This suggests that the reason behind the low ROS levels generated in F. alocis challenged neutrophils is not due to secreted bacterial products.

\section{F. alocis challenge neither primed nor inhibited neutrophil respiratory burst response}

Since the secreted bacterial products did not inhibit the respiratory burst induced by $F$. alocis challenge, I hypothesized that something expressed on the whole bacteria might be responsible for minimal generation of intracellular oxidants. To test this hypothesis, I pretreated neutrophils with media, or with F. alocis (MOI 10:1), or S. aureus (MOI 10:1) for 15 min followed by 30 min infection by $S$. aureus (MOI 10:1). I also included a co-infection condition to determine if the MOI of total bacteria made a difference in the cells response. In this "Fa $+\mathrm{Sa}$ " condition, F. alocis (MOI 5:1) and S. aureus (MOI 5:1) were added together for a total MOI of 
10 bacteria per neutrophil. F. alocis (MOI 10:1) alone was also included to show that challenged neutrophils maintain the phenotype observed in prior experiments.

Surprisingly, neither inhibition nor priming of the respiratory burst response was noticed when neutrophils were pretreated with $F$. alocis followed by S. aureus, as a second stimuli (Fig 4). The slight increase in ROS generation can be attributed to the greater total amount of bacteria per neutrophil (MOI of 20 bacteria per cell). These data suggest that $F$. alocis is not inhibiting the neutrophil respiratory burst to other stimuli. The co-infection condition $(\mathrm{Fa}+\mathrm{Sa})$ shows values that are not significantly different from the S. aureus alone condition-more like an additive effect of the response from each bacterium (Fig 4). The only significantly different condition was $F$. alocis pretreatment followed by $S$. aureus. This effect is small and is probably due to the larger overall bacterial burden experienced by the cells. My data would indicate that the oral pathogen fails to mount an oxidative response. F. alocis effectively becomes invisible to the activity of the oxidase. This shows that the failure to incite the respiratory burst is specific to F. alocis-containing phagosomes and is not a global effect within the infected neutrophil.

\section{Figure 4}

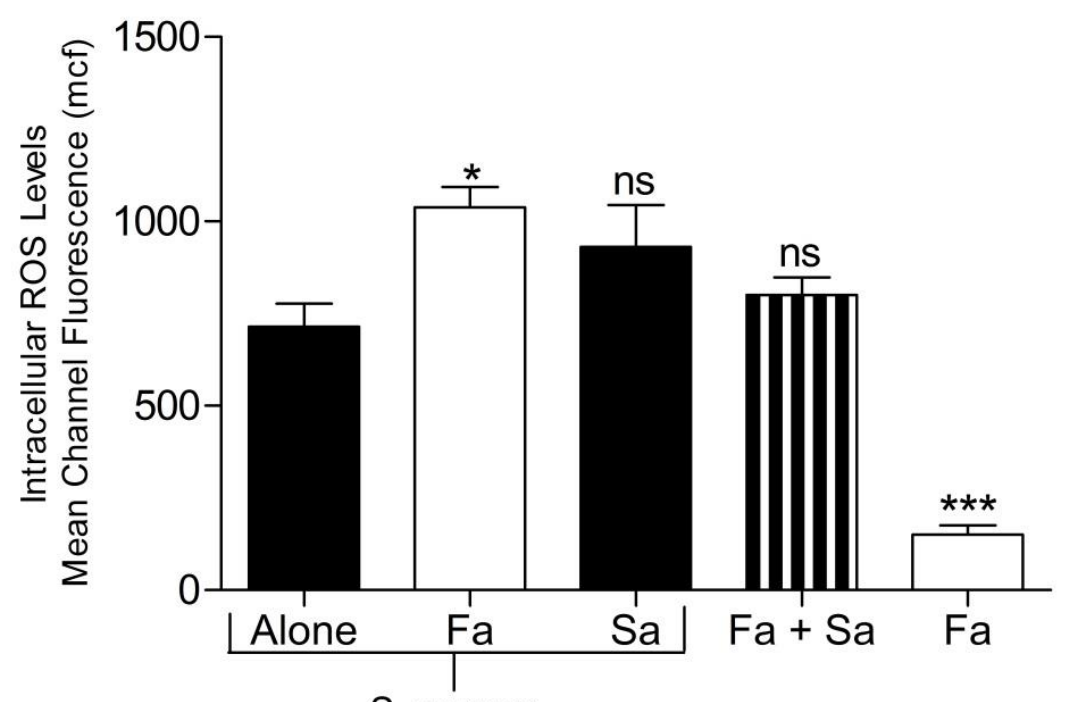

S. aureus 
Figure 4: $F$. alocis neither primes nor inhibits neutrophil respiratory burst. Neutrophils were pretreated with $F$. alocis (MOI 10:1, "Fa") or S. aureus (MOI 10:1, "Sa") for 15 min or left alone in Krebs+ buffer for 15 min (Alone), following pre-treatment all conditions were challenged with $S$. aureus (MOI 10:1, $30 \mathrm{~min}$ ) . Co-infection with $F$. alocis (MOI 5:1) and S. aureus (MOI 5:1) labeled "Fa + Sa" and F. alocis (MOI 10:1, "Fa") infections were also examined. Data are expressed as mean \pm SEM of mean channel fluorescence, $n=5$ independent experiments. All significance values are compared to $S$. aureus alone. $*=\mathbf{p}<0.05 ; * * *=\mathbf{p}<0.001$; $\mathrm{ns}=$ no significance.

\section{Viable $F$. alocis, but not heat-killed bacteria, delays specific granule recruitment to bacteria-containing phagosomes}

Upon internalization, the bacterium is contained within a membrane structure called the phagosome, whose maturation is dependent on the adequate granule fusion with it (18). The different neutrophil granule subtypes contain several microbicidal compounds in addition to membrane bound enzymes relevant to the respiratory burst response (19). After observing the very profound difference between the viable and heat-killed bacteria challenge to induce the neutrophil respiratory burst response, I decided to examine the recruitment of neutrophil granules to the bacteria-containing phagosome. I hypothesized that the reason for the absence of robust ROS generation was an inhibition of specific granule fusion to the bacteria-containing phagosome. I was interested in the specific granule fusion with the phagosome, because the membrane of this granule subtype contains $60 \%$ of the membrane bound components of the NADPH oxidase complex (gp91phox and p22phox; Table 1) which is responsible for ROS generation as previously discussed (19). Other neutrophil granules such as the secretory vesicles and gelatinase granules also contain these membrane oxidase components, but these granules are largely exocytosed and are not typically targeted to the phagosome $(19,33)$. Other bacteria have been shown to subvert granule enrichment of the phagosome to avoid killing (34). To examine if 
specific granules were recruited to $F$. alocis-containing phagosome, lactoferrin, a marker for this granule subtype, was used $(19,35)$.

Neutrophils were either untreated, which will represent a quiescent state for this cell, or cells were challenged with $S$. aureus, op-F. alocis, op-HK-F. alocis, at a MOI of 10 bacteria per cell for $30 \mathrm{~min}$. For this assay all the bacteria were labeled with a fluorochrome, to visualize them, and following stimulation cells were stained for detection of the specific granule marker and imaged by confocal microscopy. The bacteria-containing phagosome was considered positive for the granule marker if $\geq 50 \%$ of the bacterium was surrounded by it (29). Since lactoferrin is a soluble component within the granule, it will diffuse throughout the phagosome once fusion has occurred. Since there is variation between blood donors, neutrophils from one blood donor and even heterogeneity in phagosomes in a single neutrophil, the images were also quantified to ensure that the images shown herein are representative of the entire experiment. For each of three different donors, 100 infected and intact cells were quantified for lactoferrin positive and negative phagosomes. The semi-quantification expressed as percentages from the three experiments are shown in figure 5B. To control for experimenter bias, two people quantified each experiment to make sure they came to the same percentage within a 5\% margin of error. Previous studies using this method have set a cutoff of $40 \%$ or lower being necessary to determine a granule fusion failure (35). Our data showed that, 66-70\% of both S. aureus and heat-killed $F$. alocis- containing phagosomes were lactoferrin positive, whereas a significantly lower percent of $F$-alocis-containing phagosomes (35\%) were able to recruit the granule marker (Fig 5A, B). In addition, there was very little variation between the three donors as indicated by the small standard error bars. This was especially true for the viable $F$. alocis condition. One other possibility for the lower ROS values observed upon neutrophil challenge with $F$. alocis 
could have been attributed to low bacteria uptake. However, the confocal images show relatively homogenous levels of uptake among the neutrophils (Fig 5A). These results support my original hypothesis for this experiment that specific granules would not be efficiently recruited to the $F$. alocis-containing phagosomes.

\section{Figure 5}
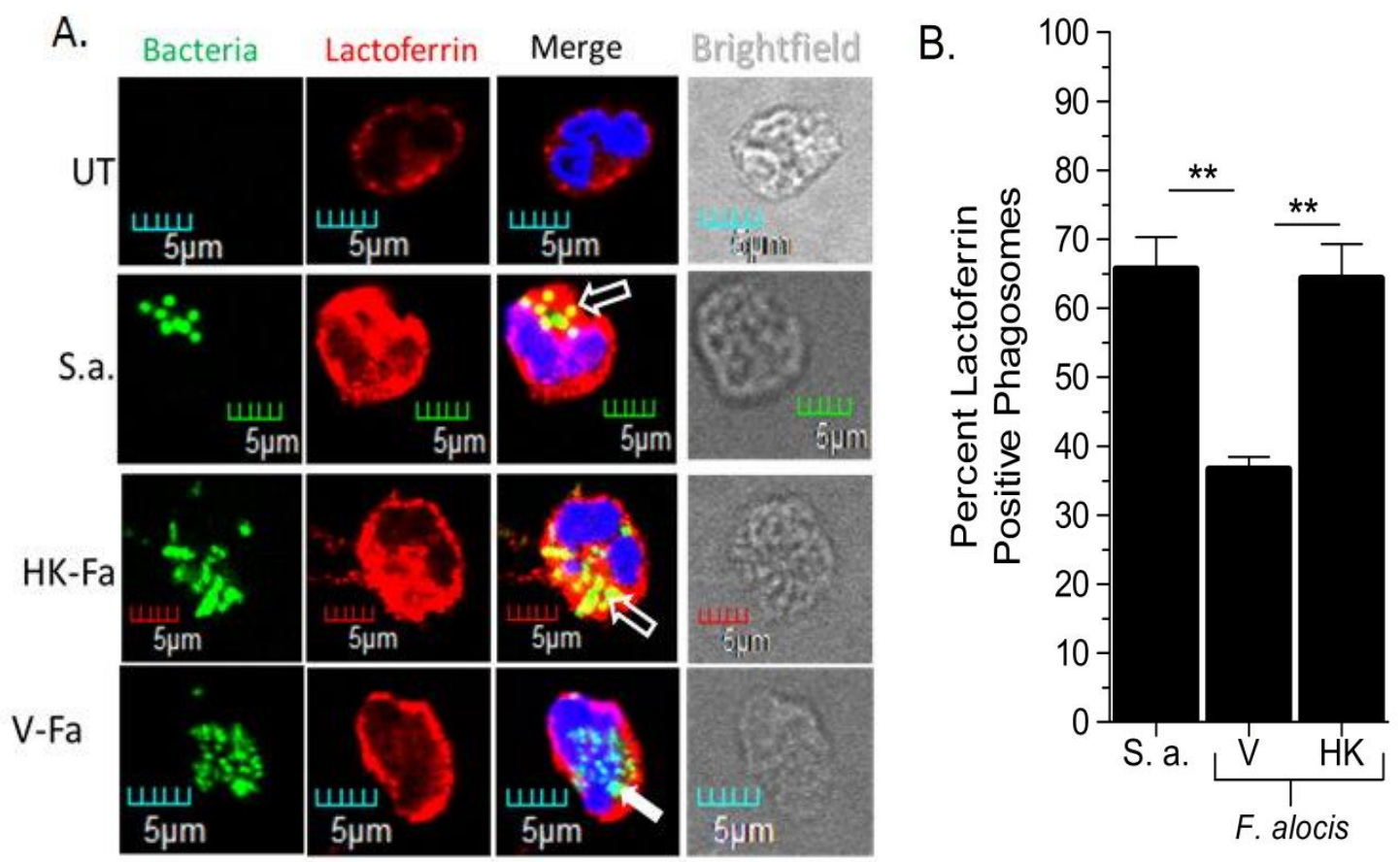

Figure 5: Phagosomes containing viable $F$. alocis are not enriched by specific granule fusion. (A) UT signifies cells that were not infected with bacteria. Human neutrophils were challenged with opsonized AF488 labeled S. aureus (S. a.) or opsonized heat-killed CFSE-labeled $F$. alocis (HK-Fa) or opsonized CFSE-labeled viable $F$. alocis (V-Fa). All bacterial challenge conditions were performed at an MOI of 10:1 with 30 min infection times. Recruitment of specific granules to bacteria-containing phagosomes was analyzed by immunostaining and subsequent confocal microscopy. In the images above, white outline arrows show lactoferrin positive phagosomes while the solid white arrows show lactoferrin negative. (B) 100 infected and intact cells for each condition were counted. For a phagosome to be considered positive, at least $\mathbf{5 0 \%}$ of the internalized bacteria must be surrounded by lactoferrin. Data are expressed as mean \pm SEM of percent of lactoferrin positive from 3 separate donors $(n=3) . * *=p<0.01$ by one-way ANOVA. 


\section{F. alocis induced secretory vesicle and specific granule exocytosis}

The results in figure 5A show that the viable $F$. alocis activates the neutrophil by moving the specific granules to the periphery of the cell instead of towards the bacteria-containing phagosome. Upon neutrophil activation, the different granule subtypes can be mobilized towards the bacteria-phagosome and/or to the cell plasma membrane and their content release to the extracellular space through a process named stimulated exocytosis. The exocytosis process is sequentially controlled with the earliest formed granules being the last to be released $(19,33)$. The earlier formed granules include the azurophil and specific granules which contain the most microbicidal and cytotoxic components (19). The later formed granules-gelatinase and secretory - are more involved in the process of chemotaxis, adhesion to the endothelium and extravasation through blood vessels to the site of infection $(19,36)$. The secretory vesicles specifically are necessary for the firm adhesion of neutrophils to the endothelial cells in the interior of the blood vessel and contain mostly albumin and other proteins of endocytic origin $(19,37,38)$.

I hypothesized that both secretory vesicles and specific granules would undergo stimulated exocytosis when cells were challenged with $F$. alocis bacteria. Neutrophils were unstimulated, or stimulated with f-Met-Leu-Phe (fMLF), a formylated bacterial peptide, used as a positive control for these experiments (26), or challenged with opsonized $F$. alocis, or heatkilled F. alocis at an MOI of 10:1 for $30 \mathrm{~min}$. Exocytosis of secretory vesicles and specific granules was determined by flow cytometry and granules identified by CD35 and CD66b respectively as previously described (26).

My data shows that $F$. alocis significantly induced secretory vesicles and specific granule exocytosis (Fig 6). This may further explain the fate of the specific granules observed in figure 
$5 \mathrm{~A}$ and why they are localized to the plasma membrane. Interestingly, the heat-killed bacteria do not show the same response as the viable bacterium in regard to the secretory vesicle exocytosis (Fig 6). The data could be interpreted to suggest that secretory vesicle exocytosis is a process actively controlled by the viable bacterium. It could also be an artifact of the experiment. Since we evaluate exocytosis by the increase on the plasma membrane expression of each granule marker, the lower CD35 expression may indicate a more rapid endocytic event of the heat-killed bacteria resulting in a false negative result for secretory vesicle exocytosis.

\section{Figure 6}

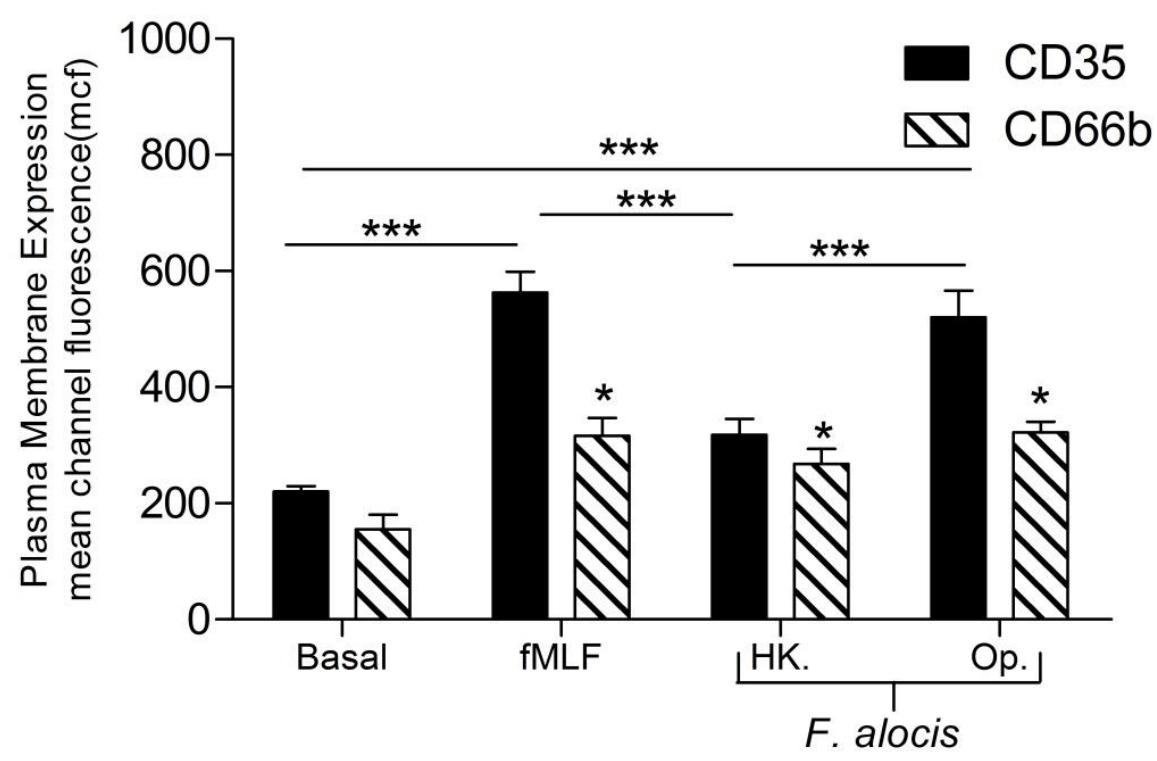

Figure 6: $F$. alocis induces secretory vesicle and specific granule exocytosis. Exocytosis of secretory vesicles and specific granules were measured as described in the methods section. Granule exocytosis of unstimulated (Basal), or fMLF stimulated (fMLF), or opsonized heat-killed $F$. alocis (HK), or opsonized viable $F$. alocis (Op) challenged cells were tested using flow cytometry. Bacterial conditions were incubated for 30 min with an MOI of 10:1. Black (CD66b) columns show levels of specific granule exocytosis while striped (CD35) columns show secretory vesicle exocytosis. Data are expressed as mean \pm SEM of mean channel of fluorescence, $n=6$ independent experiments; $* * *=p<0.001 ; *=p<0.05$ compared to basal by one-way ANOVA. 


\section{F. alocis induced minimum gelatinase granule exocytosis}

The gelatinase granule's exocytosis is critical to the extravasation of the neutrophil from the blood stream to the tissue (19). It also has been reported to carry the gp91phox and p22phox components of the NADPH oxidase (19). It is unlikely that these granules contribute directly to the observations of low ROS levels inside the cell because they are primarily exocytosed and not recruited to the phagosome $(18,19)$. However, they may play a role in priming the neutrophil (26).

F. alocis challenge showed a minimum, but statistically significant increase in gelatinase granule exocytosis when compared to basal levels (Fig 7). However, F. alocis-induced gelatinase granule exocytosis was significantly lower than the induction produced by the positive control, fMLF (Fig 7). Although neutrophil challenge with the heat-killed $F$. alocis showed a slight increase in gelatinase granule exocytosis compared to viable $F$. alocis, those differences did not achieve statistical significance. These data indicate that the bacteria viability does not play a role in the stimulation of gelatinase granule exocytosis.

\section{Figure 7}

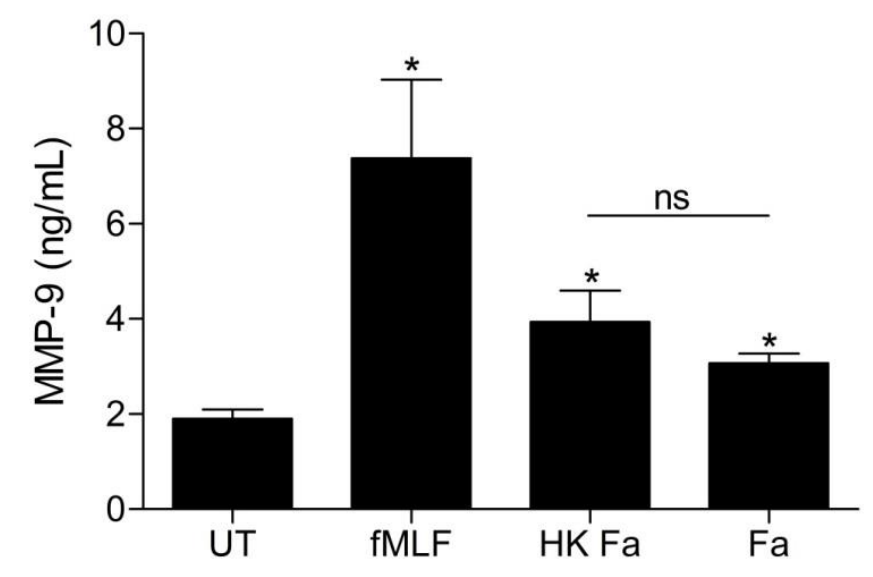

Figure 7: Both heat-killed and viable $F$. alocis induce minimum gelatinase granule exocytosis. Neutrophils were unstimulated (UT), or stimulated with fMLF (300 nM, $5 \mathrm{~min})$, or challenged with opsonized heat-killed or viable $F$. alocis 
for 30 min. All bacterial conditions used an MOI of 10:1. Samples were analyzed by ELISA as described in methods, Data are expressed as mean \pm SEM of MMP-9 release in $\mathrm{ng} / \mathrm{ml} . \mathrm{N}=6$ independent experiments; $*=\mathrm{p}<0.05$ compared to UT; ns= no significance by two-tailed Student's t-test.

\section{F. alocis does not induce azurophil granule exocytosis}

The neutrophil granule that can cause the most tissue damage if its content is release outside the cell is the azurophil granule (19). Perhaps due to the highly toxic cargo, these granules are notoriously difficult to induce to undergo exocytosis - they are primarily targeted to the phagosome $(17,33)$. These granules contain myeloperoxidase which is involved in the formation of hypochlorous acid $(\mathrm{HOCl})$ within the phagosome; therefore, it is critical to the oxidative burst response and to phagosome maturation $(17,39)$.

As a positive control, TNF- $\alpha$ followed by fMLF stimulation causes a moderate exocytosis event that is closer to physiological conditions than other reported positive controls for azurophil granule exocytosis (26). Due to the difficulty associated with mobilizing these granules, both opsonized and non-opsonized bacteria were tested. These data showed that none of the F. alocis conditions surpass the positive control and no significant difference can be seen between them and the untreated condition (Fig 8). It is possible that less uptake from the non-opsonized bacteria may increase the exocytosis. Interestingly, a significant difference was noticed between the positive control ("TNF- $\alpha+$ fMLF") and the non-opsonized conditions ("HKFa" and "Fa"). There was no significant difference between the opsonized and non-opsonized conditions however.

These data indicate that $F$. alocis challenged did not induce azurophilic granule exocytosis. Fusion of azurophil granules to bacteria-phagosome is a key event to ensure phagosome maturation and a highly toxic environment for the bacteria. It is possible that upon F. alocis challenge, azurophil granules will effectively fuse with the bacteria-phagosome unlike 
what was observed with the specific granules (Fig 5). It could also be that this granule subtype is held within the neutrophil despite ineffective granule fusion.

\section{Figure 8}

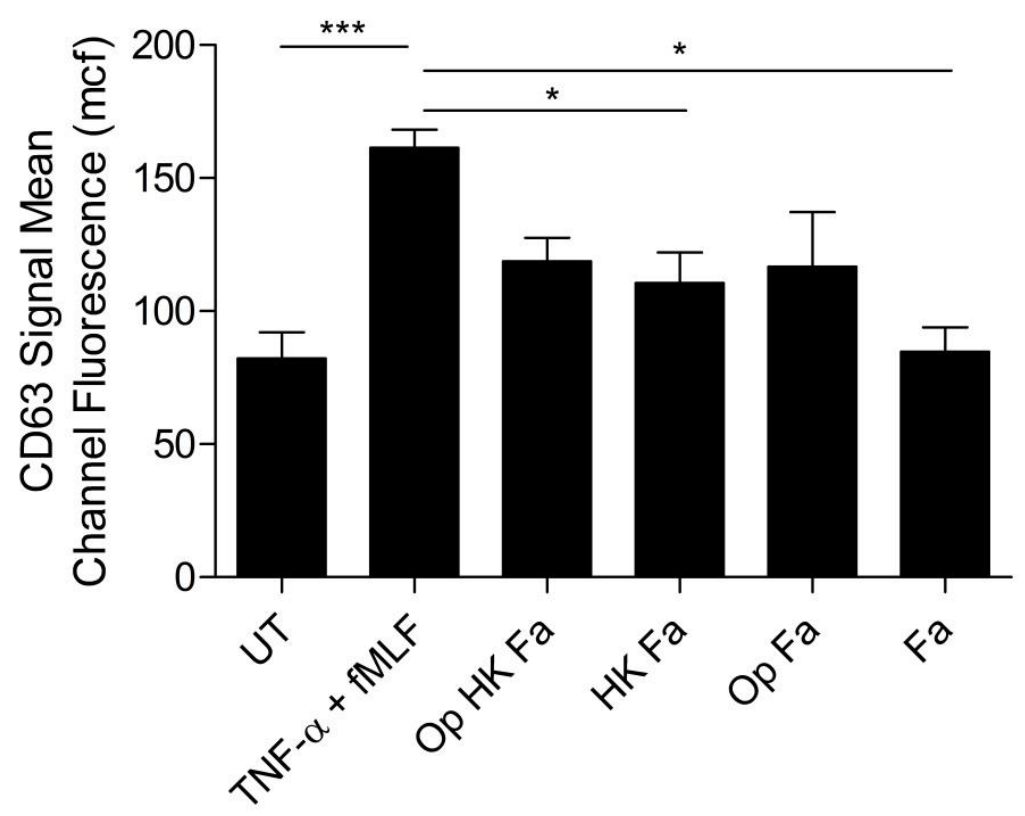

Figure 8: F. alocis does not stimulate significant release of azurophilic granules. Neutrophils were unstimulated (UT), or stimulated with TNF- $\alpha$ + fMLF, or challenged with opsonized heat-killed F. alocis (Op HK Fa) or non-opsonized heatkilled $F$. alocis (HK Fa) or opsonized F. alocis (Op Fa) or non-opsonized F. alocis (Fa). All bacterial conditions used an MOI of 10:1 for $30 \mathrm{~min}$. Azurophilic granule exocytosis was measured by the increase in plasma membrane expression of CD63 granule marker by flow cytometry. Data are expressed as mean \pm SEM of mean channel of fluorescence, $n=4$ independent experiments; $* * *=\mathbf{p}<0.01, *=\mathbf{p}<0.05$ by one-way ANOVA.

\section{Discussion}

F. alocis is only one part of the vast microbial community that exists in the oral cavity (2). It is therefore important to consider the activity of this pathogen with the activities of other periopathogens. My results show that these bacteria can induce active exocytosis (or degranulation) of three of the four neutrophil granules and can divert specific granules from the phagosome. Degranulation has been shown to be a critical step in the priming and activation of 
neutrophils (26). In the pathology of this disease, the active degranulation induced could be a strategy that these bacteria employ to recruit more neutrophils to the site of infection. $F$. alocis contains neutrophil recruiting protein A within its secretome that could be related to the observed degranulation events (40).

Previous studies have shown that $F$. alocis can also damage gingival epithelial cells on its own (41). In these studies, $F$. alocis induced the release of pro-inflammatory cytokines such as TNF- $\alpha$, IL-1 $\beta$ and IL- 6 by epithelial cells which could result in priming and/or activation of human neutrophils (41). My results show that once the neutrophils have encountered the bacteria, further degranulation events and activation occur. With the influx of cytokine signals from the epithelium and the exogenous activators from $F$. alocis, the neutrophil becomes extremely activated and many are localized to the infected periodontal pocket.

This species has shown remarkable interactions with other periodontal pathogensespecially the keystone pathogen Porphyromonas gingivalis $(11,12) . F$. alocis allows for increased growth of $P$. gingivalis in vitro (12). $P$. gingivalis also facilitates invasion of $F$. alocis into gingival epithelium (11). In its role as a pathobiont, $F$. alocis is considered to have a major impact in ameliorating the periodontal pocket environment for the other periopathogens (10). One way this has been proposed is by acting as an "oxidative sink" (10).

Neutrophils which are recruited to the periodontal pocket and subsequently the crevicular fluid form a "wall" to keep microbes from entering deeper into the tissue (14). In periodontal disease, the wall breaks down and the neutrophils are scurrying to destroy as many microbes as they can with little success (42). The crevicular fluid becomes rich in neutrophils actively secreting their granule contents and even their DNA in the process of neutrophil extracellular trap (NET) formation (21). These NETs are another weapon the neutrophil employs to detain and 
possibly kill microbes. Neutrophils eject their chromatin to ensnare microbes. The ejected DNA is studded with antimicrobial proteins such as neutrophil elastase and calprotectin $(17,43)$. The process of NETosis is usually considered to be associated with cell death (44). The neutrophils act like a kamikaze — sacrificing themselves to curb infection. However, recent evidence has shown that neutrophils undergoing the process of NET formation are still able to perform normal functions such as phagocytosis and chemotaxis (20). Unsurprisingly, the presence of NETs is highly correlated with tissue damage and autoimmune disease (45).

The exocytosis of neutrophil proteases contributes the most to the tissue damage associated with the disease. The break-up of the gingival epithelium, bleeding and bone resorption all add to the nutrient pool available to the periopathogens (46). Particularly, the exocytosis of granules increases the NADPH oxidase components on the plasma membrane which results in increased superoxide in the extracellular milieu (31). The reactive oxygen species such as superoxide alone are sufficient to damage the periodontal tissues (15). Our investigations of $F$. alocis have shown active exocytosis of the three neutrophil granules which contain NADPH oxidase components. Additionally, the secreted products of $F$. alocis are shown in this project to prime the neutrophil for an enhanced respiratory burst response. Collectively, these results indicate a possible role for $F$. alocis to increase the inflammation and tissue damage within the periodontal pocket. Interestingly, other investigations have shown that $F$. alocis is largely localized to the areas of the biofilm in contact with the soft tissue meaning that it is likely to be in direct contact with the neutrophil wall (47).

Periodontal pathogens must be able to withstand the inflammation that they provoke. $F$. alocis fails to incite a robust respiratory burst response post-phagocytosis. However, F. alocis does not inhibit the oxidase activity in the entire neutrophil. This could be a strategic method to 
keep $F$. alocis alive while allowing the neutrophil to maintain its highly activated state. This behavior will contribute both to tissue damage and may allow for the neutrophil to clear beneficial bacteria. Clearing out non-pathogenic bacteria could allow for more space and nutrients for the pathogens. Previous studies have shown that there is significantly less microbial diversity in the periodontal disease state (48). Additionally, in other anaerobic microbiota such as the gut microbiome, clearance of commensal bacteria allow for colonization by and proliferation of pathogenic bacteria (49). Such a clearance event may be modulated by periopathogens to remove competitors.

Another possible reason for $F$. alocis to prompt a less profound respiratory burst response could be to provide longevity to the neutrophils. The generation of ROS within the neutrophil results in eventual apoptosis and clearance by resident macrophages (50). This normal sequence of events prevents prolonged inflammation and tissue damage (50). Other bacterial species such as Francisella tularensis have been shown to inhibit the oxidase activity to delay apoptosiscontributing to prolonged inflammation (51). In fact, induction of neutrophil apoptosis has been proposed as a therapeutic for chronic inflammatory conditions (52).

\section{Conclusions and Summary}

F. alocis modulates neutrophil functions to activate neutrophils and contributes to the pathology of periodontitis by inducing and prolonging inflammation. $F$. alocis challenge of human neutrophils resulted in exocytosis of three of the four granule types-secretory vesicles, gelatinase and specific granules. The release of granules are associated with neutrophil recruitment (secretory and gelatinase) and tissue damage (gelatinase and specific). Granule exocytosis also contributes to neutrophil activation and priming. The failure of $F$. alocis to mount a respiratory burst response allows for increased survival of the bacteria and possibly an 
increased presence for neutrophils at the site of infection. Secreted products from F. alocis during its bacterial growth primed the neutrophil which changes the cell into a hyperactive state. Collectively, these bacterial alterations to the neutrophils result in a vicious cycle of inflammation and tissue damage which propagate each other.

\section{Figure 9}

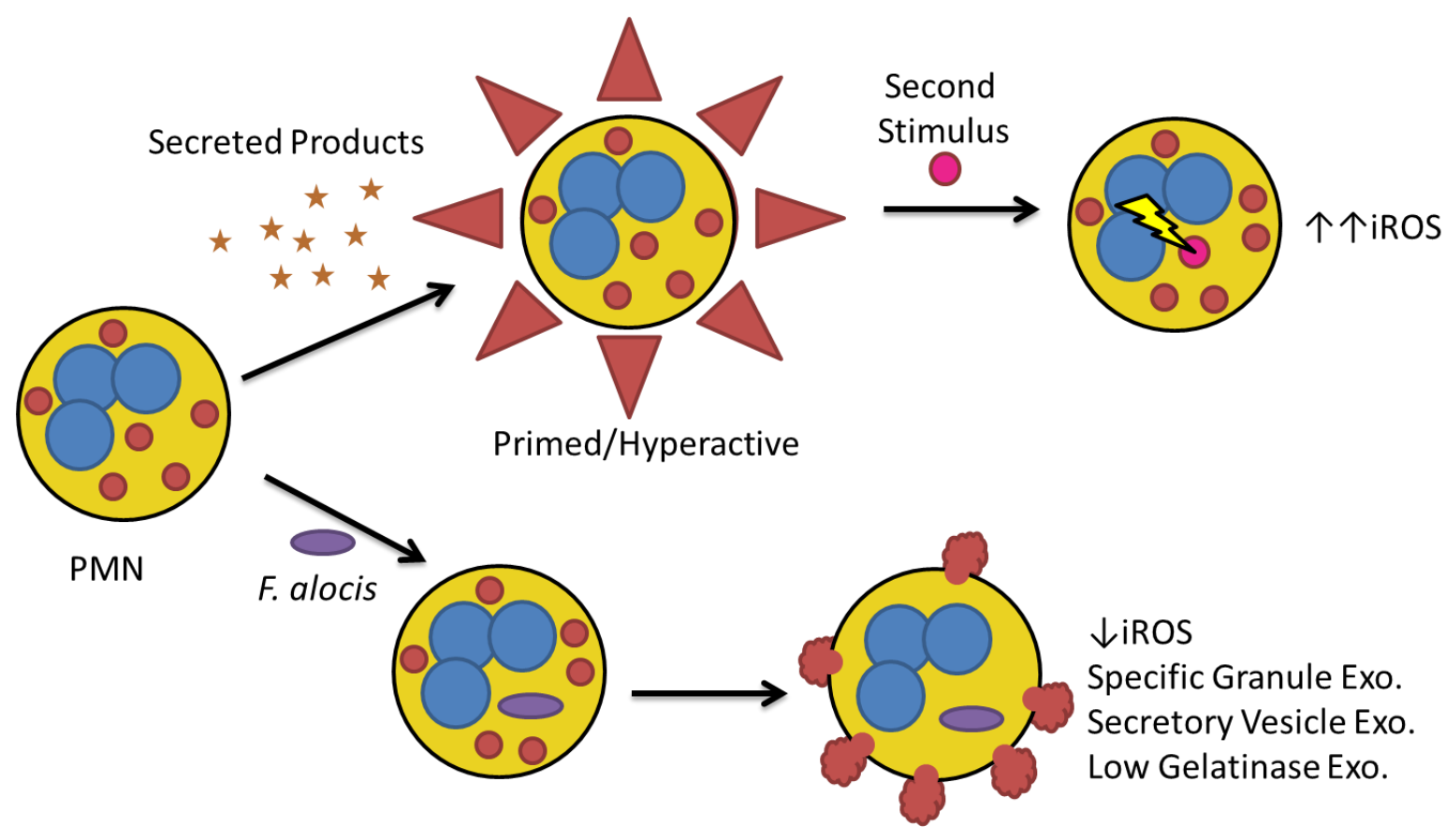

Figure 9: Model of neutrophil-F. alocis interaction. Based on the data from this study, neutrophils are able to phagocytose

F. alocis. Following internalization, there is very little ROS produced and active exocytosis of secretory vesicles, specific and gelatinase granules. When the secreted products of $F$. alocis are encountered by the neutrophil, it primes or hyperactivates the cell. A very robust generation of ROS occurs when a second stimulus is encountered.

\section{Acknowledgements}

I would like to thank Terri Manning for cell isolation and Dr. Richard Lamont for providing resources and bacteria for this project. I would also like to especially thank Cortney Armstrong, Shifu Tian and Dr. Aruna Vashishta for technical support and feedback. My highest thanks go to my mentor Dr. Silvia M. Uriarte who has provided me the opportunity to perform 
this research in addition to all her support and guidance. Funding for this project is from NIH grants DE22867 to Dr. Richard J. Lamont and DE024509 to Dr. Silvia M. Uriarte.

\section{References}

1. Hajishengallis G. 2013. Immunomicrobial pathogenesis of periodontitis: keystones, pathobionts, and host response. Trends in Immunology

2. Costalonga M, Herzberg MC. 2014. The oral microbiome and the immunobiology of periodontal disease and caries. Immunol Lett 162: 22-38

3. P.I Eke BAD, L. Wei, G.O. Thorton-Evans and R.J. Genco. 2012. Prevalence of Periodontitis in Adults in the United States. J DENT RES

4. Douglass CW. 2006. Risk assessment and management of periodontal disease. J Am Dent Assoc 137 Suppl: 27s-32s

5. Bingham CO, 3rd, Moni M. 2013. Periodontal disease and rheumatoid arthritis: the evidence accumulates for complex pathobiologic interactions. Curr Opin Rheumatol 25: 345-53

6. Kebschull M, Demmer RT, Papapanou PN. 2010. "Gum bug, leave my heart alone!"-epidemiologic and mechanistic evidence linking periodontal infections and atherosclerosis. Journal of dental research 89: 879-902

7. Hajishengallis G, Lamont RJ. 2012. Beyond the red complex and into more complexity: the polymicrobial synergy and dysbiosis (PSD) model of periodontal disease etiology. Molecular Oral Microbiology 27: 409-19

8. Hajishengallis G. 2015. Periodontitis: from microbial immune subversion to systemic inflammation. Nature Reviews Immunology 15

9. Hajishengallis G, Liang S, Payne MA, Hashim A, Jotwani R, Eskan MA, McIntosh ML, Alsam A, Kirkwood KL, Lambris JD, Darveau RP, Curtis MA. 2011. Low-abundance biofilm species orchestrates inflammatory periodontal disease through the commensal microbiota and complement. Cell Host Microbe 10: 497-506

10. Aruni WCOFHM. 2014. Filifactor alocis: The Newly Discovered Kid on the Block with Special Talents. Journal of Dental Research Journal of Dental Research

11. Aruni AW, Roy F, Fletcher HM. 2011. Filifactor alocis has virulence attributes that can enhance its persistence under oxidative stress conditions and mediate invasion of epithelial cells by porphyromonas gingivalis. Infect Immun 79: 3872-86

12. Wang Q, Wright CJ, Dingming H, Uriarte SM, Lamont RJ. 2013. Oral Community Interactions of Filifactor alocis In Vitro. PLOS ONE 8: e76271

13. Wang Q, Jotwani R, Le J, Krauss JL, Potempa J, Coventry SC, Uriarte SM, Lamont RJ. 2014. Filifactor alocis infection and inflammatory responses in the mouse subcutaneous chamber model. Infect Immun 82: 1205-12

14. Ryder MI. 2010. Comparison of neutrophil functions in aggressive and chronic periodontitis. Periodontology 2000 53: 124-37

15. Scott DA, Krauss JL. 2012. Neutrophils in periodontal inflammation. Frontiers of Oral Biology 15: 56-83 
16. Kolaczkowska E, Kubes P. 2013. Neutrophil recruitment and function in health and inflammation. Nat Rev Immunol 13: 159-75

17. Amulic B, Cazalet C, Hayes GL, Metzler KD, Zychlinsky A. 2012. Neutrophil function: from mechanisms to disease. Annu Rev Immunol 30: 459-89

18. Nordenfelt P, Tapper H. 2011. Phagosome dynamics during phagocytosis by neutrophils. J Leukoc Biol 90: 271-84

19. Borregaard N, Sørensen OE, Theilgaard-Mönch K. 2007. Neutrophil granules: a library of innate immunity proteins. Trends in Immunology 28: 340-5

20. Yipp BG, Petri B, Salina D, Jenne CN, Scott BN, Zbytnuik LD, Pittman K, Asaduzzaman M, Wu K, Meijndert HC, Malawista SE, de Boisfleury Chevance A, Zhang K, Conly J, Kubes P. 2012. Infection-induced NETosis is a dynamic process involving neutrophil multitasking in vivo. Nat Med 18: 1386-93

21. Vitkov L, Klappacher M, Hannig M, Krautgartner WD. 2010. Neutrophil fate in gingival crevicular fluid. Ultrastruct Pathol 34: 25-30

22. Allen LA. 2003. Mechanisms of pathogenesis: evasion of killing by polymorphonuclear leukocytes. Microbes Infect 5: 1329-35

23. Hurst JK. 2012. What really happens in the neutrophil phagosome? Free Radic Biol Med 53: $508-20$

24. Devlin TM. 2011. Textbook of biochemistry : with clinical correlations. Hoboken, NJ: John Wiley \& Sons

25. Allen LA. 2006. Pivotal Advance: Francisella tularensis LVS evades killing by human neutrophils via inhibition of the respiratory burst and phagosome escape. Interview by Helene F. Rosenberg. J Leukoc Biol 80: 1222-3

26. Uriarte SM, Rane MJ, Luerman GC, Barati MT, Ward RA, Nauseef WM, McLeish KR. 2011. Granule exocytosis contributes to priming and activation of the human neutrophil respiratory burst. J Immunol 187: 391-400

27. Dupre-Crochet S, Erard M, Nubetae O. 2013. ROS production in phagocytes: why, when, and where? J Leukoc Biol 94: 657-70

28. Allen LA. 2007. Immunofluorescence and confocal microscopy of neutrophils. Methods Mol Biol 412: 273-87

29. Jog NR, Rane MJ, Lominadze G, Luerman GC, Ward RA, McLeish KR. 2007. The actin cytoskeleton regulates exocytosis of all neutrophil granule subsets. Am J Physiol Cell Physiol 292: C1690-700

30. McLeish KR, Uriarte SM, Tandon S, Creed TM, Le J, Ward RA. 2013. Exocytosis of neutrophil granule subsets and activation of prolyl isomerase 1 are required for respiratory burst priming. J Innate Immun 5: 277-89

31. Kruger P, Saffarzadeh M, Weber AN, Rieber N, Radsak M, von Bernuth H, Benarafa C, Roos D, Skokowa J, Hartl D. 2015. Neutrophils: Between host defence, immune modulation, and tissue injury. PLoS Pathog 11: e1004651

32. Condliffe AM, Kitchen E, Chilvers ER. 1998. Neutrophil priming: pathophysiological consequences and underlying mechanisms. Clin Sci (Lond) 94: 461-71

33. Sengelov H, Kjeldsen L, Borregaard N. 1993. Control of exocytosis in early neutrophil activation. J Immunol 150: 1535-43

34. Staali L, Bauer S, Morgelin M, Bjorck L, Tapper H. 2006. Streptococcus pyogenes bacteria modulate membrane traffic in human neutrophils and selectively inhibit azurophilic granule fusion with phagosomes. Cell Microbiol 8: 690-703 
35. Allen LA, Beecher BR, Lynch JT, Rohner OV, Wittine LM. 2005. Helicobacter pylori disrupts NADPH oxidase targeting in human neutrophils to induce extracellular superoxide release. J Immunol 174: 3658-67

36. Borregaard N, Kjeldsen L, Sengelov H, Diamond MS, Springer TA, Anderson HC, Kishimoto TK, Bainton DF. 1994. Changes in subcellular localization and surface expression of L-selectin, alkaline phosphatase, and Mac-1 in human neutrophils during stimulation with inflammatory mediators. J Leukoc Biol 56: 80-7

37. Borregaard N, Kjeldsen L, Rygaard K, Bastholm L, Nielsen MH, Sengel $\varnothing v$ H, Bjerrum OW, Johnsen AH. 1992. Stimulus-dependent secretion of plasma proteins from human neutrophils. Journal of Clinical Investigation 90: 86-96

38. Uriarte SM, Powell DW, Luerman GC, Merchant ML, Cummins TD, Jog NR, Ward RA, McLeish KR. 2008. Comparison of proteins expressed on secretory vesicle membranes and plasma membranes of human neutrophils. J Immunol 180: 5575-81

39. 1988. The dynamics of exocytosis in human neutrophils. The Journal of Cell Biology 107: $2117-23$

40. Aruni AW, Roy F, Sandberg L, Fletcher HM. 2012. Proteome variation among Filifactor alocis strains. Proteomics 12: 3343-64

41. Moffatt CE, Whitmore SE, Griffen AL, Leys EJ, Lamont RJ. 2011. Filifactor alocis interactions with gingival epithelial cells. Molecular oral microbiology 26: 365-73

42. Hajishengallis G, Lamont RJ. 2014. Breaking bad: manipulation of the host response by Porphyromonas gingivalis. Eur J Immunol 44: 328-38

43. Urban CF, Ermert D, Schmid M, Abu-Abed U, Goosmann C, Nacken W, Brinkmann V, Jungblut PR, Zychlinsky A. 2009. Neutrophil extracellular traps contain calprotectin, a cytosolic protein complex involved in host defense against Candida albicans. PLoS Pathog 5: e1000639

44. Remijsen Q, Kuijpers TW, Wirawan E, Lippens S, Vandenabeele P, Vanden Berghe T. 2011. Dying for a cause: NETosis, mechanisms behind an antimicrobial cell death modality. Cell Death and Differentiation 18: 581-8

45. Kaplan MJ, Radic M. 2012. Neutrophil extracellular traps: double-edged swords of innate immunity. J Immunol 189: 2689-95

46. Nussbaum G, Shapira L. 2011. How has neutrophil research improved our understanding of periodontal pathogenesis? Journal of Clinical Periodontology 38: 49-59

47. Schlafer S, Riep B, Griffen AL, Petrich A, Hübner J, Berning M, Friedmann A, Göbel UB, Moter A. 2010. Filifactor alocis - involvement in periodontal biofilms. BMC Microbiology 10: 66-

48. Wang J, Gao Y, Zhao F. 2015. Phage-bacteria interaction network in human oral microbiome. Environ Microbiol

49. Khanna S, Pardi DS. 2016. Clinical implications of antibiotic impact on gastrointestinal microbiota and Clostridium difficile Infection. Expert Rev Gastroenterol Hepatol

50. Fox S, Leitch AE, Duffin R, Haslett C, Rossi AG. 2010. Neutrophil apoptosis: relevance to the innate immune response and inflammatory disease. J Innate Immun 2: 216-27

51. Schwartz JT, Barker JH, Kaufman J, Fayram DC, McCracken JM, Allen LA. 2012. Francisella tularensis inhibits the intrinsic and extrinsic pathways to delay constitutive apoptosis and prolong human neutrophil lifespan. J Immunol 188: 3351-63

52. Hampson P, Hazeldine J, Lord JM. 2013. Neutrophil apoptosis and its induction as a potential treatment for chronic inflammatory disease. Curr Opin Hematol 20: 10-5 